\title{
Evaluation of Soft-Tissue Model Parameters
}

\author{
Matthias Teschner, Sabine Girod, and Kevin Montgomery \\ National Biocomputation Center \\ Stanford University, Palo Alto, CA 94304, USA \\ teschner@stanford.edu
}

\begin{abstract}
Computer-based techniques for the simulation of craniofacial surgical procedures and for the prediction of the surgical outcome have been shown to be very useful. However, the assessment of the accuracy of the simulated surgical outcome is difficult. In this paper, a robust registration technique is described which allows to compare the simulated surgical outcome and the actual surgical result.
\end{abstract}

\section{Methods}

In order to simulate craniofacial surgical procedures an existing system is used which is based on a preoperative CT scan of the patient's head and on a preoperative surface scan of the patient's face. The simulated postoperative patient's appearance is compared to a second surface scan which is obtained postoperatively. To enable the comparison, the pre- and postoperative surface scan have to be registered. Registration is required due to the fact, that the pre- and the postoperative scan are most probably obtained with different positions of the patient's head relative to the scanner.

In contrast to the Iterative Closest Point algorithm, which minimizes the mean of the Euclidean distances between corresponding points, a robust registration method is employed, which minimizes the median of the Euclidean distances of corresponding points. This is due to the fact, that the pre- and the postoperative facial scan differ in certain areas, i. e., corresponding points in these areas will still have comparative large Euclidean distances. In case of minimizing the median error instead of the mean error, these points do not falsify the transformation computed by the registration process.

If both scans are registered, the surgery simulation is performed using the preoperative scan of the patient's face and the simulation result is compared to the actual postoperative appearance by assessing the differences of corresponding points. Parameters of the soft-tissue model, such as number of soft-tissue layers or spring constants, are adapted with respect to minimized differences of corresponding points of the simulated and the actual surface of a patient's face.

\section{Results}

Registration of pre- and postoperative surface scans provides the opportunity to assess the accuracy of the simulation result by comparing the simulated and 

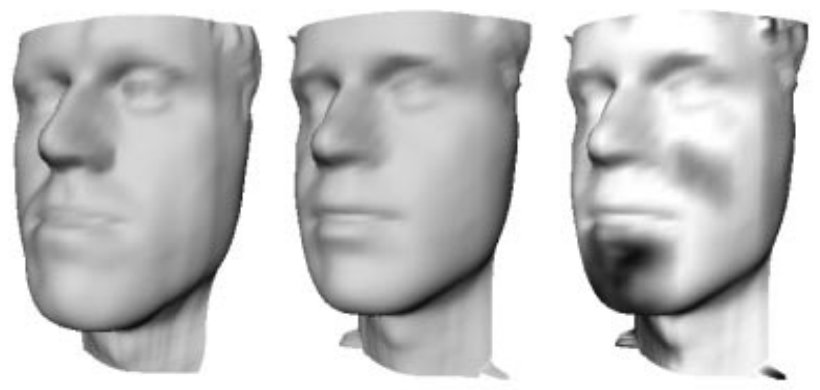

Fig. 1. Left: Praeoperative surface scan. Middle: Postoperative surface scan. Right: Registration error mapped onto the postoperative surface scan. Dark areas represent a large Hausdorff-distance up to $6 \mathrm{~mm}$. Bright areas are regions with no significant registration error.

the actual postoperative patient's appearance. Differences between both scans can be minimized by the adaption of model parameters. Fig. 1 1 shows a pre- and postoperative surface scan of a patient's face. Both scans are registered and the registration error is visualized in the right-hand image. Dark colors represent large errors (Hausdorff distances up to $6 \mathrm{~mm}$ ). Areas influenced by the surgery show large registration errors. However, these areas do not falsify the registration of the entire scan. Fig. 2 shows two scans with and without swelling. This swelling can be seen in the right-hand image which visualizes the registration error of these two scans. It can be seen that the registration approach is not influenced by areas which are different in both scans. The registration shown in Fig. 1 can be used to compare the simulated postoperative patient's appearance and his actual postoperative appearance. The registration shown in Fig. 2 can be employed to objectively assess the swelling, e. g. by measuring the volume of the swelling.
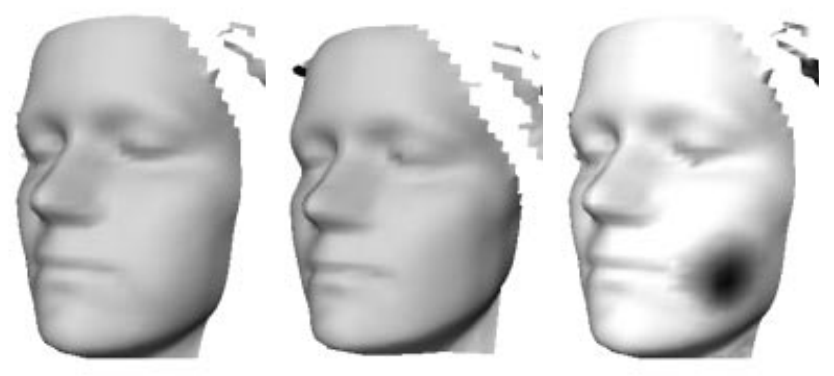

Fig. 2. Left: Surface scan with swelling. Middle: Surface scan without swelling. Right: Registration error mapped onto the surface scan with swelling. Dark areas represent a large Hausdorff-distance up to $4 \mathrm{~mm}$. Bright areas are regions with no significant registration error. 